\section{Encountering COVID in a Cancer Ward: Lessons in Infection Prevention}

The coronavirus disease (COVID-19) pandemic has brought in unique issues for healthcare workers (HCW) managing health services of non-COVID patients [1]. This infection poses a double-edged sword while managing immuno-compromised patients [2]. As these individuals are at an increased risk of mortality from infections, most centers treating cancer have reduced hospital visits, modified chemotherapy protocols and rely heavily on tele-consultations [3]. The other major concern that has emerged while managing these children is the safety of healthcare workers (HCW) and sustainability of the nonCOVID treatment centers.

We recently managed a child with acute lymphoblastic leukemia (ALL) who was subsequently diagnosed as being COVID positive. On examination in our daycare, she was afebrile, mildly tachypneic (respiratory rate of 25 breaths $/ \mathrm{min}$ ) with saturation of $88-89 \%$ in room air, and bilateral consolidation in lower lobes on $X$-ray chest. Although acute leukemia presenting with pneumonia is not unusual, the presence of hypoxia in room air was more suggestive of viral/ pneumocystis etiology. She was shifted to the COVID isolation ward without admission in the cancer ward. On evaluation, she was detected to be RT-PCR positive for severe acute respiratory syndrome coronavirus 2 (SARS-COV-2) and was managed as per standard guidelines. She recovered and was discharged after few weeks. Bone marrow evaluation confirmed ALL and she was started on treatment which is presently ongoing.

All HCWs in our team had been on hydroxychloroquine prophylaxis as per Indian Council of Medical Research (ICMR) advisory, since 2-3 weeks prior to this exposure [4]. On analysis of the exposure of healthcare staff to this patient, only six were high-risk and rest were low-risk [5]. The three primary nurses who initially interacted with her were quarantined from the day of the exposure. All HCWs who managed her, except the initial four, used appropriate personal protective equipment (PPE) [5]. The patient and her attendant wore triple-layer mask on hospital entry and all doctors and nurses in the team used N95 masks. All contacts were tested between 5-14 days of exposure and were negative. The services of the department were temporarily discontinued for a period of 14 days from last exposure to the contact. The patients admitted in the same ward and their attendants were observed for a period of 14 days from exposure and were asymptomatic. The daycare area was sanitized as per protocol on the same day after shifting the patient.
We wish to highlight certain issues related to containing COVID-19 infection in a non-COVID facility:

Non COVID facilities- low risk or high risk? In hospitals catering to both COVID-19 and non-COVID patients, albeit in separate buildings, COVID areas are considered high-risk, and non-COVID areas aree considered low risk. As per the MOHFW initial and revised guidelines on rational use of PPE, wards and ICUs in non-COVID areas continue to be marked low-risk [6.7]. As majority of COVID-19 positive in our country have been asymptomatic, it is only logical that many patients/attendants we encounter in non-COVID areas may be carriers of the virus, thus placing all the HCW in these areas at risk of infection. Thus the demarcation between low- and highrisk areas is no longer absolute. All HCW encountering patients (COVID or non-COVID) should take utmost precautions for personal protection to reduce exposure to the virus.

PPE rationalization: In resource-limited settings, rationalizing PPE use to ensure adequate supply to those HCW working in COVID hospitals and to prevent misuse, seems judicious [8]. However, without adequate PPE, accidental encounter with patients places $\mathrm{HCW}$ to high-risk category of exposure with mandatory quarantine of entire units, thus resulting in closure of treatment centers. Additionally, there is a bigger risk of exposure of other patients in the same facility, which could be more dangerous. Hence, when non-COVID areas are made functional in hospitals, adequate supply of PPE needs to be ensured for all HCWs.

Holding area in non-COVID hospitals: The recommendation is to develop a common holding area (after screening for COVID suspects) for non-COVID pa tients coming for routine treatment came later [8]. These patients could either be treated and sent home from this area itself or be tested for COVID and admitted to their respective wards after negative report is confirmed [8].

Universal testing of non-COVID patients: Although universal testing of patients who come for non-COVID treatment may be a safe strategy for healthcare workers [9], it does raise some concerns. Unlike in government facilities, most private laboratories charge between Rs 3500-4500 per RTPCR test. The turnaround time is usually 24 hours, which makes it difficult to get this test before providing acute treatment. For patients who need non-COVID treatment regularly such as those on dialysis, chemotherapy, blood transfusions etc, it will not be feasible to do tests before every hospital admission. The revised ICMR guidelines also do not recommend testing of asymptomatic individuals [10]. Although, real time PCR is the gold standard for diagnosis of this infection, false negative reports are known to occur, especially following early testing, improper collection and sample transport and primer related concerns [11]. In suspected COVID patients with corroborative symptoms, a repeat test is recommended. However, in the 
current argument for mandatory testing in non-COVID patients, a proportion of patients may thus be missed despite testing.

As India transitions into a 'living with COVID' strategy, we will be encountering more of these patients in non-COVID settings. As patients and HCW are equally at risk of lifethreatening complications of COVID infections, all efforts must still be made to protect all from getting infected. Also, HCW need to adapt themselves and work with each patient and attendant as if they are encountering a potential COVID carrier with universal precautions, appropriate PPE and standard steps for infection prevention. Another helpful strategy would be to develop teams of HCWs with 1-2 weeks of work followed by two weeks of quarantine, as is followed for COVID areas, even for non-COVID areas. In the event of an accidental exposure, this will prevent shutting down of services due to quarantining of staff.

Above all, a positive frame of mind is of utmost importance to tide over this difficult phase of for us and patients alike.

Published online: June 09, 2020; PII: S097475591600189

Nita Radhakrishnan* and SaVITRI Singh Department of Pediatric Hematology Oncology, Super Speciality Pediatric Hospital and PG Teaching Institute, Noida, Uttar Pradesh, India

\section{REFERENCES}

*nitaradhakrishnan@yahoo.com

1. Ministry of health and Family Welfare, Government of India. Enabling Delivery of Essential Health Services during the COVID 19 Outbreak: Guidance note. Available from: https://www.mohfw.gov.in/pdf/ Essential services during COVID19 updated 0411201.pdf. Accessed May 14, 2020.

2. Terenziani M, Massimino M, Biassoni V, Casanova M, Chiaravalli S, Ferrari A, et al. SARS-CoV-2 disease and children under treatment for cancer. Pediatr Blood Cancer. 2020; 28346.

3. Seth R. COVID -19 pandemic: The challenges for pediatric oncology. Indian Pediatr. 2020 May 4 [Early online]. Available from: https://www.indianpediatrics.net/COVID 29.03.2020/CORR-00171.pdf. Accessed June 7, 2020.

4. Ministry of health and Family Welfare, Government of India. Advisory on the use of hydroxyl chloroquine as prophylaxis for SARS-COV2 Infection. Available from: https://www.mohfw.gov.in/pdf/Advisory on the use of Hydroxychloroquin asprophylaxis for SARS CoV2 infection.pdf. Accessed May 14, 2020.

5. Director General Medical Education, Uttar Pradesh. Quarantine policy document for the health care workers (HCWS) working in COVID hospital/ward. Available from: http://gsvmmedicalcollege.com/wp-content/uploads/2020/ 05/Quarantine-policy-DGME-4.4.2020.pdf. Accessed May 14, 2020.

6. World Health Organization. Health workers exposure risk assessment and management in the context of COVID-19 virus: Interim guidance, 4 March, 2020. Available from: https://apps.who.int/iris/ handle/10665/331340. Accessed May 14, 2020.

7. Ministry of Health and Family Welfare, Government of India. Novel Coronavirus Disease 2019 (COVID-19): Guidelines on rational use of Personal Protective Equipment (setting approach for Health functionaries working in nonCOVID areas). Available from: https://www.mohfw.gov. in/pdf/GuidelinesonrationaluseofPersonalProtective Equipment.pdf. Accessed May 14, 2020.

8. Ministry of Health and Family Welfare, Government of India. Novel Coronavirus Disease 2019 (COVID-19): Additional guidelines on rational use of Personal Protective Equipment (setting approach for Health functionaries working in non-COVID areas). Available from:https:// www.mohfw.gov.in/pdf/Additionalguidelinesonrational use ofPersonalProtectiveEquipment settingapproachforHealth functionariesworkinginnon COVIDareas.pdf. Accessed May 14, 2020.

9. ESMO guideline: Cancer patient management during the COVID-19 pandemic. Available from: https:// www.esmo.org/guidelines/cancer-patient-managementduring-the-covid-19-pandemic. Accessed May 14, 2020.

10. ICMR Strategy for COVID19 testing in India (Version 4, dated 09/04/2020) Available from: https://main.icmr.nic. in/ sites/default/ files/ upload_documents/ Strategey_for COVID19_Test_v4_09042020.pdf. Accessed May 14, 2020.

11. Tahamtan A, Ardebili A. Real-time RT-PCR in COVID-19 detection: Issues affecting the results. Expert Rev Mol Diagn. 2020;20:453-4.

\section{Seasonal Influenza Vaccination and the Heightened Risk of Coronavirus and Other Pandemic Virus Infections: Fact or Fiction?}

During this ongoing severe acute respiratory illness coronavirus 2 (SARS-CoV-2) pandemic, few speculative reports on significant association of influenza vaccines with an increased risk of coronavirus infection appeared both in media and academic circles. The speculation of vaccines paradoxically increasing the risk of infections possibly originated first following 2009 influenza A (H1N1pdm09) pandemic when four Canadian studies suggested that receipt of seasonal influenza vaccine increased the risk of laboratory-confirmed 2009 pandemic influenza A (H1N1pdm09) virus infection [1]. This led to five additional studies, each of which substantiated these initial findings. One proposed mechanism behind this phenomenon is 'original antigenic sin' which was first used to describe how first exposure to influenza virus shapes the outcome of subsequent exposures to antigenically related strains. When an individual is 